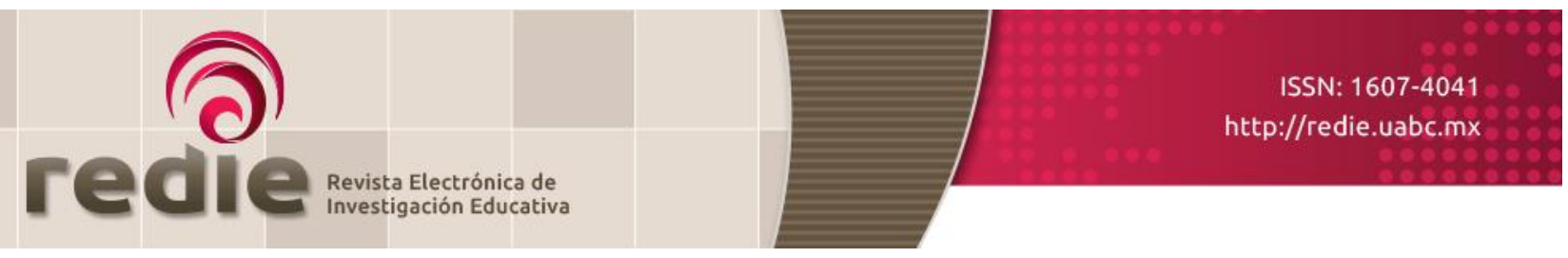

Vol. 20, Núm. 3, 2018

\title{
Ética de la investigación y formadores de docentes ${ }^{1}$ Research Ethics and Teacher Trainers
}

\author{
Ana Hirsch Adler (1) anaha007@yahoo.com.mx \\ Cecilia Navia Antezana (2) ceeeci@yahoo.com \\ (1) Universidad Nacional Autónoma de México \\ (2) Universidad Pedagógica Nacional \\ (Recibido: 5 de enero de 2017; Aceptado para su publicación: 4 de noviembre de 2017)
}

Cómo citar: Hirsch, A. y Navia, C. (2018). Ética de la investigación y formadores de docentes. Revista Electrónica de Investigación Educativa, 20(3), 1-10. https://doi.org/10.24320/redie.2018.20.3.1776

\section{Resumen}

El presente artículo busca articular un enfoque particular de la ética de la investigación (Human Research Ethics) con respecto a las prácticas de investigación que realizan los formadores de docentes y sus estudiantes en comunidades de México y Bolivia. Se presentan los elementos teóricos de la ética de la investigación y las categorías generadas con base en las respuestas de 79 formadores de docentes a la pregunta abierta sobre los factores de protección que se deben tomar en cuenta con respecto a los participantes de las investigaciones. La pregunta forma parte de un cuestionario cualitativo aplicado entre marzo y junio de 2015. La principal conclusión es que aunque la función sustantiva que desempeñan estos profesores es la preparación de nuevos maestros de educación básica, al indagar sobre los factores de protección logran enunciar con claridad aspectos centrales de la ética de investigación.

Palabras clave: Ética, ética del docente, formador de docentes, vulnerabilidad.

\section{Abstract}

This paper seeks to construct a particular approach to human research ethics with respect to research practices carried out by teacher trainers and their students in communities in Mexico and Bolivia. It presents the theoretical elements of research ethics and the categories generated on the basis of answers from 79 teacher trainers to an open question on protective factors that should be considered with regard to study participants. The question is part of a qualitative questionnaire administered between March and June 2015. The study concludes that when asked about protective factors, teachers are able to clearly state key aspects of research ethics, although their core focus is on training new basiclevel education teachers.

Keywords: Ethics, teachers responsibility, teacher educators, vulnerability.

\footnotetext{
${ }^{1}$ Este artículo es producto de dos proyectos de investigación: Excelencia del Profesorado Universitario de Posgrado de la Universidad Nacional Autónoma de México, aprobado por el Programa de Apoyo a Proyectos de Investigación e Innovación Tecnológica (IN300217) y Ética Profesional en la Formación de Profesores, desarrollado en la upN-Unidad Ajusco. En ambos, los temas de ética profesional docente y ética de la investigación son prioritarios.
} 


\section{Introducción}

El objetivo del artículo consiste en articular un enfoque particular de la ética de la investigación referido a los seres humanos (Human Research Ethics), con respecto a las prácticas de investigación que llevan a cabo los formadores de docentes y sus estudiantes en comunidades en condición de vulnerabilidad en dos países latinoamericanos: México y Bolivia.

Es importante señalar que la ética de la investigación con seres humanos proviene originalmente -y ha sido desarrollada- por las Ciencias de la Salud; en este trabajo se busca conocer y repensar esta propuesta con el fin de explorar procesos sociales y educativos.

En el apartado a continuación se presentan elementos teóricos centrales sobre la ética de la investigación cuando se lleva a cabo con seres humanos. El punto principal de este enfoque radica en la necesidad de proteger los derechos y el bienestar de los participantes, buscando al mismo tiempo la construcción de nuevo conocimiento y que pueda resultar de beneficio tanto para los sujetos bajo estudio como para los investigadores (Shamoo \& Resnik, 2009). Se incluyen los objetivos de esta aproximación ética y los principios acordados por la mayor parte de los autores de este campo temático.

En el segundo apartado se hace el análisis de las respuestas que dieron 79 formadores de docentes (56 en México y 23 en Bolivia) a una pregunta abierta que explora los factores de protección que los formadores de docentes consideran necesario tener en cuenta para realizar investigación con comunidades en condiciones de vulnerabilidad. Para el análisis se construyeron categorías, subcategorías y rasgos que combinan los elementos teóricos y lo encontrado en las instituciones formadoras de docentes.

\subsection{Elementos teóricos de la ética de la investigación con seres humanos}

La perspectiva Human Research Ethics se origina y emplea fundamentalmente en las Ciencias de la Salud, pero por su importancia ha ido permeando otras áreas de conocimiento. De acuerdo con Walton (2016) hay tres objetivos en la ética de la investigación: 1) proteger a los participantes, 2) asegurar que la investigación se conduzca de manera que sirva a los intereses individuales, de grupo y de la sociedad, y 3) evaluar las actividades específicas de investigación buscando su solidez ética en relación con el manejo del riesgo, la protección de la confidencialidad y el consentimiento informado.

Las normas éticas en la investigación, de acuerdo con Resnik (2016), promueven: los fines de la investigación -principalmente el conocimiento y la verdad-; los valores esenciales para el trabajo colaborativo, como son: confianza, rendición de cuentas, respeto mutuo y trato justo; la construcción del apoyo público y los valores éticos y sociales, tales como: responsabilidad, derechos humanos, cumplimiento de la legalidad, salud pública y seguridad.

Los principios éticos generales que se aplican en este tipo de investigación son: Autonomía, Beneficencia y Justicia (The National Commission for the Protection of Human Subjects of Biomedical and Behavioral Research, 1979), en dicho documento, Autonomía -o respeto por las personas- se refiere al derecho de los individuos de determinar en cuáles actividades de investigación van a participar y en cuáles no. Se requiere que sean capaces de entender qué es lo que les solicitan, llevar a cabo un juicio razonado sobre el efecto que tendrá para ellos y hacerlo libremente sin influencias coercitivas. Para ello, en el Código de Núremberg (1947) se plantea el proceso del consentimiento informado y se incorporan dos convicciones éticas: los individuos deben ser tratados como agentes autónomos y las personas con autonomía disminuida tienen derecho a mayor protección. Shamoo y Resnik (2009) consideran que los sujetos vulnerables son aquellos que carecen de las capacidades necesarias para tomar decisiones o de poder para promover sus propios intereses. Consideramos que en el desarrollo de las investigaciones tendrían que considerarse las desigualdades existentes en las comunidades, de modo que se pueda evitar la imposición de dicho ejercicio de poder, por parte de los investigadores, cuando estos son portadores de una visión ajena a la cultura y a sus necesidades. 
La investigación repercute no sólo en los participantes, sino también en las comunidades donde ésta se lleva a cabo [...]. Los investigadores deben participar activamente con las comunidades en la toma de decisiones acerca del diseño y la realización de la investigación [...] siendo sensibles y respetuosos hacia las prácticas culturales, tradicionales y religiosas de las comunidades (Organización Mundial de la Salud y Organización Panamericana de la Salud, 2012, p. 15).

La condición de vulnerabilidad no implica que las personas, grupos y comunidades se excluyan de las investigaciones que puedan beneficiarlos, sino que es necesario adecuar el diseño de la investigación de modo que no se generen situaciones que les produzcan daño (National Bioethics Advisory Commission, 2001).

Una buena manera de proceder para conocer e intervenir en las comunidades es considerar los factores de riesgo que pueden ser responsables de un problema en particular. Dichos factores son aspectos de una persona o grupo, de su contexto o experiencia que hacen más o menos probable que vayan a experimentar un determinado problema. Entre los factores de protección personales que pueden contrarrestar los de riesgo están, por ejemplo, conocimiento y habilidades y experiencia e historia, y los del contexto se refieren a las condiciones en que viven las personas (Work Group for Community Health and Development at the University of Kansas, 2016).

La Beneficencia se refiere a la obligación del investigador de maximizar los beneficios y minimizar los riesgos de daño a los individuos y a la sociedad. Beauchamp y Childress (2001) separan este principio en dos: Beneficencia y No Maleficencia, este último consiste en mantener continua vigilancia para que los participantes no reciban daño alguno, puesto que se tiene la responsabilidad de asegurar su bienestar físico, emocional y social. La No Maleficencia resultó de particular interés para el análisis de la información obtenida en el trabajo de campo.

En el enfoque Human Research Ethics la Justicia se refiere a la equidad en la distribución entre cargas y beneficios. Exige que estos últimos no se utilicen únicamente por quienes pueden utilizarlos y que no implique indebidamente a personas y grupos que no serán beneficiados. De acuerdo con Australian Government (2016) el "Principio de Justicia" trata de: la justa distribución de los beneficios y riesgos, el justo tratamiento del reclutamiento de los participantes y la revisión de la investigación por parte de comités especializados.

La aceptabilidad ética de la investigación tiene que ver con un índice aceptable de beneficios potenciales frente a los riesgos de daño, la minimización de los riesgos, los procedimientos adecuados de consentimiento informado [...] las medidas para garantizar la protección de las poblaciones vulnerables, los procedimientos justos para la selección de participantes y la consideración de la repercusión de la investigación sobre las comunidades de donde proceden los participantes, tanto durante la investigación como después de que ésta finalice (Organización Mundial de la Salud y Organización Panamericana de la Salud, 2012, p. 12).

En torno a los principios existen algunas reglas. Una de las más importantes en este enfoque es la de la confidencialidad. De acuerdo con Matthew y Sutton (2011) esta regla ética se refiere a que los datos personales del sujeto investigado no se expondrán en el reporte de la investigación, pues es indispensable proteger al informante de posibles daños.

\section{Método}

Se diseñó un cuestionario cualitativo con diez preguntas abiertas, que se aplicó entre marzo y junio de 2015 a 79 formadores de docentes (56 en México y 23 en Bolivia) encargados de la preparación de jóvenes interesados en dedicarse a la docencia en educación básica o de maestros en servicio que requerían contar con una licenciatura.

La información se obtuvo en las siguientes instituciones, en México: Instituto Superior de Ciencias de la Educación del Estado de México (Unidad Tejupilco), Escuela Normal de Santa Ana Zicatecoyan, Escuela 
Normal de Atlacomulco "Profesora Evangelina Alcántara Díaz" del Estado de México, y el Centro Regional de Educación Normal "Benito Juárez", del Estado de Hidalgo; y en Bolivia: Instituto Superior de Formación de Maestros "Simón Bolívar" y Escuela Normal de Warisata, ambos del Departamento de La Paz.

Cuatro de estas instituciones (tres en México y una en Bolivia) se ubican en zonas rurales, mientras que dos se localizan en áreas urbanas. Todas siguen un modelo de formación inicial de maestros que integra elementos de tipo pedagógico y educativo, así como actividades de investigación. Esto incluye, por lo general, el desarrollo de diagnósticos educativos y socioeducativos en las escuelas, así como diferentes modalidades de intervención y de investigación participativa en las comunidades.

Si bien en las instituciones en las que se realizó este estudio tanto los formadores de docentes como los estudiantes consideran importante llevar a cabo procesos de investigación, el trabajo que realizan se centra fundamentalmente en la docencia. Ambas funciones sustantivas están articuladas, puesto que el trabajo de campo es parte de su proceso formativo. Los mismos principios, reglas y valores pueden encontrarse tanto en la ética de la docencia como en la ética de la investigación y, sin embargo, cada una de estas éticas aplicadas pueden presentarse de maneras particulares e interpretarse de formas diversas.

Del cuestionario de 10 preguntas abiertas se seleccionó la siguiente: ¿Qué factores de protección considera que deben tener en cuenta los investigadores cuando trabajan con comunidades que se encuentran en condición de vulnerabilidad? ${ }^{2}$

El número de respuestas obtenidas en las instituciones formadoras de docentes en México fue de 140 y en Bolivia de 58. Esta diferencia entre los dos países se debe a que el cuestionario cualitativo se aplicó en más centros formadores de docentes en México y por ello se obtuvo información más diversificada.

El procedimiento de análisis consistió en capturar $^{3}$ de forma escrita todo lo expresado por los sujetos de la investigación y clasificarlo por temas recurrentes y significativos. Como ya se mencionó, se tomaron en cuenta los aspectos del marco teórico; con base en todas las categorías se derivaron subcategorías y rasgos, para dicha construcción se tomó en cuenta la propuesta de Coffey y Atkinson (2003), que consistió en identificar temas y patrones clave, condensando el grueso de los datos en unidades analizables para crear con ellos (o a partir de ellos) las categorías. Todas las respuestas se integraron en tres grandes rubros: Principios, reglas y valores; Factores de protección y Profesionalismo.

\footnotetext{
${ }^{2}$ Las otras preguntas son: En términos generales, indique los que a su juicio son las principales características de "ser un profesor excelente"; Mencione cinco acciones para la formación en valores profesionales en los docentes; Describa una acción que haya realizado con sus alumnos que considere que haya contribuido a su formación como profesionales éticos; ¿Cuáles han sido los cinco obstáculos más importantes a los que se ha enfrentado en la formación de profesionales éticos?; ¿Qué postura ética considera usted que debe tener un investigador cuando los sujetos en estudio pertenecen a grupos en situación de vulnerabilidad?; ¿Qué responsabilidad adquiere el investigador cuando trabaja con personas, grupos y comunidades en situación de vulnerabilidad; ¿Cuáles cree usted que son los principales rasgos de un profesor que trabaja con grupos vulnerables? y ¿Qué beneficios considera usted que puede proporcionar la investigación a la población en estudio?

${ }^{3}$ La captura se realizó con el programa Word.
} 


\section{Discusión}

En las respuestas de los participantes de ambos países se encontraron aspectos ligados a los principios y reglas de la investigación formulados en el campo teórico y en diferentes instrumentos normativos, así como un marco de valores centrado en el respeto (ver tabla I).

Tabla I. Categoría Principios, reglas y valores

\begin{tabular}{l|l}
\hline Subcategorías & Rasgos \\
\hline \multirow{2}{*}{ Principios } & No Maleficencia \\
& Beneficencia \\
\hline \multirow{2}{*}{ Reglas } & Autonomía \\
\hline Valores & Confidencialidad \\
Veracidad \\
\hline
\end{tabular}

Fuente: Elaboración propia.

\subsection{Principios y reglas}

Retomamos la propuesta de Beauchamp y Childress (2001), que como se indicó antes, proponen cuatro principios: Beneficencia, No Maleficencia, Autonomía y Justicia, a diferencia del Informe Belmont (The National Commission for the Protection of Human Subjects of Biomedical and Behavioral Research, 1979) que integra en uno solo los dos primeros.

En el trabajo empírico se encontraron menciones sobre tres de ellos: No Maleficencia, Beneficencia y Autonomía, mientras que el principio de Justicia no fue mencionado por los participantes.

Sobre el principio de No Maleficencia, se hizo referencia a la necesidad de evitar el daño y minimizar los riesgos con respecto a todas las personas involucradas en los procesos de investigación. En el caso de las instituciones educativas mexicanas en las que se aplicó el cuestionario, se indicaron los siguientes asuntos: no provocar cambios descontextualizados, no imponer puntos de vista, evitar que cuando los investigadores finalizan su trabajo los participantes no se sientan abandonados, no abusar de la naturaleza bondadosa, no actuar con fines de lucro, evitar palabras y gestos que ofendan, no herir susceptibilidades, no dañar en sus investigaciones a segundas o terceras personas, evitar prejuicios y no hacerlos sentir mal. Con respecto a las respuestas de Bolivia, encontramos únicamente tres: no denigrar a las personas y comunitarios, no discriminar y evitar prejuicios. Como puede verse, en ambos países está presente la idea de evitar el daño.

Sobre el principio de Beneficencia, en México, los encuestados expresaron la necesidad de: brindar apoyo, cuidar a los involucrados, que la investigación contribuya a mejorar sus condiciones, procurar el bienestar y apoyo al grupo, tener una percepción positiva de los participantes y proteger la dignidad humana. Las respuestas emitidas por los formadores de docentes enfatizan el papel que ellos deben desempeñar en las comunidades a donde llevan a sus alumnos, sin que hayan señalado la parte que debe corresponder a los integrantes de las comunidades en estudio.

En Bolivia sólo se hizo referencia a generar procesos de reflexión comunitaria, lo que puede significar que se insiste en que la investigación debe compartirse entre los investigadores y los investigados.

En cuanto al principio de Autonomía y a la obligación de cumplir con el consentimiento informado, en México se dijo lo siguiente: autonomía en general, necesidad de proporcionar datos esenciales, consentimiento informado en general, establecer los propósitos de manera explícita y mostrar los resultados. Mientras que en Bolivia sólo se hizo mención a la autoestima.

De las cuatro reglas que plantean Beauchamp y Childress (2001), que son: confidencialidad, veracidad, privacidad y fidelidad, aparecieron sólo las dos primeras. En México, sobre la confidencialidad, se enunció 
la importancia de la protección de la identidad de los participantes, no revelar la confidencialidad, brindar confianza para promover la comunicación, mantener el anonimato, proteger los datos y ser discreto. En Bolivia se afirmó la confidencialidad y confianza y el manejo de documentos. En ambos países, se planteó la veracidad con respecto a hablar con la verdad, no mentir, no alterar los datos y no plantear hallazgos incorrectos.

\subsection{Valores}

El valor más importante en el enfoque Human Research Ethics es el respeto, lo cual coincide en los resultados obtenidos tanto en México como en Bolivia. En el trabajo de campo aparece en relación con diferentes aspectos; uno de ellos es el contexto, que tiene un peso muy fuerte en el ejercicio de la investigación e integra temas como: respeto por la cultura, ideología, idiosincrasia, usos y costumbres y condiciones de los grupos con los que se desarrolla la investigación.

Los formadores de docentes consideraron que tomar en cuenta este valor puede evitar la discriminación y asegurar el éxito de los procesos. Se refirieron específicamente al vínculo de los investigadores (formadores de docentes y sus estudiantes) con los participantes de las comunidades, con el propósito de ganar su confianza y no imponer sus puntos de vista, manifestando actitudes de respeto, humildad e igualdad. En el caso de México, los valores que se mencionaron (además del respeto) son: valores en general, honestidad, humildad, tolerancia, igualdad y responsabilidad. En el caso de Bolivia se indicaron los siguientes: compromiso, solidaridad, templanza, prudencia, humildad e igualdad.

\subsection{Factores de protección}

Los formadores de docentes distinguieron dos tipos de factores de protección: de los participantes de las investigaciones y de los investigadores (ver tabla II).

Tabla II. Factores de protección

\begin{tabular}{c|l}
\hline \multicolumn{1}{c|}{ Subcategorías } & \multicolumn{1}{c}{ Rasgos } \\
\hline \multirow{3}{*}{ Para los participantes } & $\begin{array}{l}\text { Reconocimiento del contexto } \\
\text { Trato sensible } \\
\text { Medidas de protección }\end{array}$ \\
\hline \multirow{3}{*}{ Para los investigadores } & $\begin{array}{l}\text { Manejar de manera adecuada el involucramiento } \\
\text { con los participantes } \\
\text { Procesos de gestión } \\
\text { Trabajo en contextos de inseguridad }\end{array}$ \\
\hline
\end{tabular}

Fuente: Elaboración propia.

Factores de protección para los participantes de las investigaciones. Se definieron tres subcategorías: reconocimiento del contexto, trato sensible y medidas de prevención.

En cuanto al primer aspecto, en México, se señalaron los siguientes temas: conocer el contexto familiar, la situación de las personas mayores, los aspectos sociales, culturales, políticos y educativos y documentarse sobre el tipo de personas y comunidades. De manera coincidente, en Bolivia se expresó el requerimiento de caracterizar a las comunidades y a su cultura.

Las ideas relacionadas con un trato sensible hacen referencia a la calidad humana que hay que tener con respecto a los participantes. Se presentaron ideas sobre: la necesidad de introducirse al grupo de manera paulatina para que no se incomoden por la presencia de los investigadores y que las personas que trabajen directamente con personas y comunidades en condición de vulnerabilidad deben actuar con mucho cuidado, de tal manera que no hieran las susceptibilidades de los informantes. Entre los rasgos que consideran que deben tener los investigadores están, en México: sencillez, amabilidad, empatía, prudencia y sensibilidad, y en Bolivia se enfatizaron los rasgos de ser reservado, seguro, diplomático y cuidadoso. 
En México, se indicaron como medidas de prevención: definir un inventario de riesgos, que el investigador evalúe el grado de afectación de los participantes para determinar el nivel de riesgo y los factores de protección necesarios, no involucrarse tanto con la comunidad, no presentarse con instrumentos que evidencien la situación en la que vive el grupo, evitar los factores de vulnerabilidad, involucrarse en estrategias participativas de investigación, usar diferentes instancias de apoyo, tener claridad acerca de qué es lo que se desea lograr y cuál es el procedimiento y establecer reglas entre investigadores e investigados. Bolivia mencionó: seguir las normas institucionales, tener prevención, potenciar el desarrollo personal y promover el trabajo comunitario.

Factores de protección para los investigadores. Se ubicó como factor de protección el ser intermediario entre la población y las autoridades. Sobre los factores de riesgo se manifestaron: no saber manejar de manera adecuada el involucramiento con las personas, grupos y comunidades en situación de vulnerabilidad y el trabajo que se desarrolla en contextos de inseguridad. Sorprendentemente, este último sólo se indicó en Bolivia.

\subsection{Profesonalidad}

Concentramos todas las respuestas acerca del ejercicio ético de los formadores de docentes y sus alumnos, en dos subcategorías: el ejercicio profesional ético del investigador y sus habilidades de comunicación (tabla III).

Tabla III. Profesionalidad

\begin{tabular}{l|l}
\hline Subcategorías & Rasgos \\
\hline \multirow{2}{*}{ Ejercicio profesional ético } & Conocimiento de capacidades \\
& $\begin{array}{l}\text { Ser muy profesional } \\
\text { Actitudes }\end{array}$ \\
\hline Habilidades de comunicación & $\begin{array}{l}\text { Necesidad del manejo de un lenguaje sensible } \\
\text { Fomentar el trabajo en equipo }\end{array}$ \\
\hline
\end{tabular}

Fuente: Elaboración propia.

Con respecto a la primera subcategoría, en México se indicaron los siguientes asuntos: conocimiento de las capacidades cognitivas y metodológicas del propio investigador, hacer estudios de caso semejantes, ser muy profesional al cuestionar, ser prudente y tener tacto en la aplicación de los instrumentos.

Por otro lado, se expresó directamente que se requiere de la ética profesional para no afectar a los grupos investigados y atender la diversidad de situaciones que se pueden encontrar en el desarrollo del trabajo. También se mencionaron actitudes, tales como: dedicación, seguridad en lo que se va a hacer y seriedad en la investigación. En Bolivia, de manera semejante, se hizo referencia a hacer bien la investigación, tener ética profesional y contar con actitudes de seriedad, seguridad y apertura.

La segunda subcategoría se refiere a las habilidades de comunicación. En México se manifestó la necesidad del manejo de un vocabulario accesible y de fomentar el trabajo en equipo; mientras que en Bolivia se hizo mención de la comunicación en general, así como del trabajo comunitario y el carácter de complementariedad.

\section{Conclusiones}

Uno de los principales aportes de este trabajo radica en que cuando se les preguntó a los formadores de docentes sobre los factores de protección que se deben promover con las personas, grupos y comunidades que participan en las actividades de investigación, en ambos países pudieron dar cuenta, con sus propias palabras, de diferentes aspectos de la ética de la investigación.

De cierta forma no sorprende que los aspectos éticos, tales como principios, reglas y valores sean los más señalados por los profesores que contestaron el cuestionario, pues la pregunta se refiere directamente a 
los factores de protección, que se ubica claramente en la ética de la investigación, pero habíamos supuesto que no tendrían un conocimiento especializado en torno a esta temática. Sin embargo, encontramos en sus respuestas una mayor diversificación de ideas respecto a los elementos que componen la ética profesional, así como la presencia de términos específicos, tales como: confidencialidad, decir la verdad, no hacer daño, beneficiar a las comunidades y consentimiento informado. Resultó también significativo que hubo numerosas respuestas acerca de la necesidad de profesionalismo y de un ejercicio profesional de carácter ético.

En las categorías que se construyeron con respecto a los factores de protección se pudo hacer una diferencia entre las medidas de protección para los sujetos de la investigación y para los investigadores. La mayoría de los trabajos sobre la ética de la investigación tiende a favorecer la protección de los participantes y, sin embargo, es igualmente importante considerar la de los investigadores.

En la categoría de factores de protección para los participantes, como ya se mencionó en la descripción de los resultados, los tres rasgos son: reconocimiento del contexto, trato sensible y medidas de protección. En el caso de los investigadores, los rasgos fueron: manejar de manera adecuada el involucramiento con los participantes, los procesos de gestión y el trabajo en contextos de inseguridad. Este último fue señalado únicamente en el caso de Bolivia, y consideramos que particularmente en el caso de México, la inseguridad que se está produciendo en el país afecta severamente a los investigadores.

Se encontró en las respuestas de los participantes un vínculo interesante con una línea de investigación que se dedica a las consideraciones éticas sobre la vulnerabilidad en la investigación cualitativa (Hammersley y Traianou, 2014; Loue, Comité Central de Bioética y Molina, 2015). Se prioriza en esta corriente la necesidad de conocer el contexto, con el fin de definir si la comunidad en donde se propone realizar el estudio, se encuentra en condiciones de vulnerabilidad. Los formadores de docentes señalaron cuestiones similares a lo planteado por los autores, tanto en la importancia del contexto, como en que la investigación debe contribuir a mejorar las condiciones de las personas y comunidades.

Es evidente que, especialmente cuando se trata de poblaciones en condición de vulnerabilidad, debe llevarse a cabo un acercamiento con los líderes y personas respetadas por los sujetos. También lo es considerar el peso que tienen las autoridades políticas, administrativas y educativas en sus distintos niveles: nacional, regional y local. Entre los asuntos mencionados para apoyarlos, se manifestó que los maestros en formación podrían fungir como mediadores con las autoridades. Indicaron también que podían contribuir a detectar necesidades y problemas, así como a formular posibles soluciones para resolverlos.

En otro asunto, con base en las respuestas de los formadores de docentes, se hizo evidente la tensión entre dos posturas: una mayoritaria que apunta al respeto de las comunidades y de su cultura, y otra minoritaria que indicó la necesidad de intervenir para cambiar las condiciones en que viven que, en algunos casos, se podría inferir como una acción intrusiva a sus culturas. Por ello, consideramos que parte del trabajo que requieren realizar los formadores con respecto a sus estudiantes consiste en dialogar con ellos sobre la complejidad de los procesos sociales y acerca de los límites de lo que puede hacerse para mejorar las condiciones de vida de los pobladores.

En el mismo sentido, es importante recuperar un aspecto sobre el cual ha habido insistencia durante décadas, sobre todo en las Ciencias Sociales y Humanas, que es el de la necesidad de devolver la información a los participantes. Esto puede llevarse a cabo, por ejemplo, ampliando y difundiendo el conocimiento alcanzado para que los sujetos en estudio puedan hacer uso de él para su propio beneficio.

En términos comparativos, se encontró que los formadores de docentes de ambos países reconocen la importancia de considerar en sus prácticas -así como en los procesos formativos de sus estudiantesaspectos ligados a la ética de la investigación cuando ésta se realiza con personas o comunidades. Sin embargo, encontramos algunas diferencias que pueden explicarse por el contexto sociopolítico y cultural de ambos países. 
En los formadores de Bolivia existe la preocupación por proteger a los participantes de las investigaciones de la discriminación y los prejuicios, así como de considerar la práctica de la investigación con un sentido de horizontalidad y confianza con los participantes. En México se tiende a privilegiar más aspectos del buen ejercicio profesional, así como la posibilidad de apoyar como mediadores con instituciones y autoridades locales. Preocupa el hecho de que si bien en Bolivia se habla de la importancia de dar seguridad a los investigadores cuando realizan trabajo de campo; en México, país que vive problemas importantes de seguridad, ese tema no fue mencionado.

\section{Referencias}

Australian Government, National Health and Medical Research Council (2016). Section 1: Values and principles of ethical conduct and chapter 2.1 Risk and benefit. Recuperado de https://msd.unimelb.edu.au/sites/default/files/docs/e72_national_statement_march_2014_140331.pdf

Beauchamp, T. y Childress, J. (2001). Principles of biomedical ethics (5a. ed.). Nueva York: Oxford University Press.

Código de Núremberg (1989). Ética médica (J. A. Mainetti, trad.). Argentina. (Obra original publicada en 1947). Recuperado de http://www.bioeticanet.info/documentos/Nuremberg.pdf

Coffey, A. y Atkinson, P. (2003). Encontrar el sentido a los datos cualitativos. Estrategias complementarias de investigación. Colombia: Universidad de Antioquia.

Hammersley, M. y Traianou, A. (2014). An alternative ethics? Justice and care as guiding principles for qualitative research. Sociological Research Online, 19(3), 24.

Loue, S., Comité Central de Bioética y Molina, D. P. (2015). Las consideraciones éticas sobre la vulnerabilidad en la investigación cualitativa. Revista Facultad Nacional de Salud Pública, 33. Recuperado de http://aprendeenlinea.udea.edu.co/revistas/index.php/fnsp/article/view/24545/20779953

Matthew, D. y Sutton, C. (2011). Being ethical. En D. Matthew y C. Sutton (Eds.), Social research. An introduction (pp. 29-53). Londres: Sage.

National Bioethics Advisory Commission (2001). Ethical and policy issues in research involving human participation (Vol. 1). Recuperado de https://bioethicsarchive.georgetown.edu/nbac/human/overvol1.pdf

Organización Mundial de la Salud y Organización Panamericana de la Salud (2012). Capítulo III Pautas y orientación dirigidas a los miembros de los comités de ética de la investigación. Pautas y orientación operativa para la revisión ética de la investigación en salud con seres humanos, pp. 11-16. Recuperado de http://www.paho.org/hq/+index.php?option=com docman\&task=doc view\&gid=22738\&ltemid=270

Resnik, D. (2016). What is Ethics in Research \& Why is it important? National Institute of Environmental Health Sciences. Recuperado de https://www.niehs.nih.gov/research/resources/bioethics/whatis/

Shamoo, A. y Resnik, D. (2009). Responsible conduct of research. Nueva York: Oxford University Press.

The National Commission for the Protection of Human Subjects of Biomedical and Behavioral Research, Office of the Secretary (1979). The Belmont Report. Ethical Principles and Guidelines for the Protection of Human Subjects of Research. Recuperado de https://videocast.nih.gov/pdf/ohrp belmont report.pdf

Walton, N. (2016). What is Research Ethics? Recuperado de https://researchethics.ca/what-is-researchethics/ 
Work Group for Community Health and Development at the University of Kansas (2016). Chapter 19, Section 2. Understanding risk and protective factors: their use in selecting potential targets and promising strategies for intervention. Recuperado de http://ctb.ku.edu/en/table-of-contents/analyze/ choose-and-adapt-community-interventions/risk-and-protective-factors/main 